\title{
A space $C(K)$ where all nontrivial complemented subspaces have big densities
}

\author{
by \\ Piotr Koszmider (São Paulo)
}

\begin{abstract}
Using the method of forcing we prove that consistently there is a Banach space (of continuous functions on a totally disconnected compact Hausdorff space) of density $\kappa$ bigger than the continuum where all operators are multiplications by a continuous function plus a weakly compact operator and which has no infinite-dimensional complemented subspaces of density continuum or smaller. In particular no separable infinitedimensional subspace has a complemented superspace of density continuum or smaller, consistently answering a question of Johnson and Lindenstrauss of 1974.
\end{abstract}

1. Introduction. Let us start the paper by some quotations from a paper of Johnson and Lindenstrauss ([JL]):

... it was shown in [AL] that if $X$ is WCG and $Y$ is a separable space of $X$ then there is a separable $Z$ with $X \supset Z \supset Y$ such that $Z$ is complemented in $X$. It is well known that for non WCG spaces $X$ this assertion may fail to be true. For example, $l_{\infty}$ has no complemented infinite-dimensional and separable subspaces. Pełczyński raised the question whether $l_{\infty}$ is the worst example in the sense that whenever $X \supset Y$, with $Y$ separable, there is a $Z$ so that $X \supset Z \supset Y$, $Z$ complemented in $X$ and $Z$ isomorphic to a subspace of $l_{\infty}$. Example 1 shows that this is not the case....

Let $X \supset Y$ be Banach spaces, with $Y$ separable. Does there exist a space $Z$ with $X \supset Z \supset Y, Z$ complemented in $X$ and the density character of $Z$ is less than or equal to that of the continuum?

Assuming special properties of the Banach space in question the positive answer to the above question has been obtained: the results of S. Heinrich and P. Mankiewicz ([HM]) imply it for dual spaces, S. Gul'ko ([Gu]) proved

2000 Mathematics Subject Classification: 03E35, 46B03.

While conducting the research leading to the results presented in this paper, the author was partially supported by a fellowship Produtividade em Pesquisa from National Research Council of Brazil (Conselho Nacional de Pesquisa, Processo Número 300369/01-8) and by the State of São Paulo Research Assistance Foundation (Fundação de Amparo á Pesquisa do Estado de São Paulo, Processo Número 02/03677-7). Part of this research was done while the author was visiting the Technical University of Łódź which partially supported the author as well. We are thankful to Prof. Marek Balcerzak for organizing the visit. 
it for $C(K)$ where $K$ is extremally disconnected, and A. Plichko [Pl] for spaces whose dual balls are weak* angelic. $[\mathrm{PY}]$ contains a recent survey on related questions where the following general two-cardinal property $\mathcal{C P}(\lambda, \kappa)$ of a Banach space $X$ is considered: every subspace of $X$ of density character at most $\lambda$ is contained in a complemented subspace with density character at most $\kappa$.

The main result of this paper is that the negative answer to the question of Johnson and Lindenstrauss is consistent $\left({ }^{1}\right)$ even in the class of Banach spaces of continuous functions on compact Hausdorff spaces.

We prove that $\mathcal{C P}(\omega, \kappa)$ may be false for any $\kappa<2^{\omega_{1}}$ in the presence of the continuum hypothesis, i.e., $2^{\omega}=\omega_{1}$ (abbreviated $\mathrm{CH}$ in the sequel) together with an arbitrarily big value of $2^{\omega_{1}}$ on the scale of alephs, i.e., $\mathcal{C P}\left(\omega,\left(2^{\omega}\right)^{+}\right), \mathcal{C P}\left(\omega,\left(2^{\omega}\right)^{++}\right)$etc. are consistently false as well. Actually our spaces $C(K)$ have much stronger properties which may be interesting by themselves: they are of density $2^{\omega_{1}}$ but they have no infinite-dimensional complemented subspaces of density $\kappa<2^{\omega_{1}}$, and any linear operator defined on them is of the form $g I+S$, where $g \in C(K)$ and $S$ is weakly compact (or equivalently strictly singular).

It remains open if the counterexample can be obtained in ZFC or if $\mathcal{C P}\left(\omega, 2^{\omega_{1}}\right)$ holds for any Banach space. We do not even know if $\mathcal{C P}(\omega, \kappa)$ holds for all Banach spaces for any $\kappa$ like for example a measurable or a supercompact cardinal.

The construction is a stepping-up of the construction from [Ko] where new constructions of Banach spaces with few operators and so with few complemented subspaces were presented. It is done by forcing (see $[\mathrm{Ku}]$ ), however, it seems that it can be done by using $\mathrm{CH}$ and the principles developed in [Ve1] which are true in Gödel's constructible universe $L$. This line has a potential (see [Ve2]) in moving on the scale of beths and providing counterexamples to $\mathcal{C P}\left(\omega, 2^{2^{\omega}}\right), \mathcal{C P}\left(\omega, 2^{2^{2^{\omega}}}\right)$ etc. It also suggests that, assuming $\mathrm{CH}$, large cardinals (in the sense like in [Ka]) may be necessary to prove the consistency of statements like: $\mathrm{CH}+$ Every Banach space of continuous functions on a compact Hausdorff space of density $\omega_{2}$ has a complemented subspace of density $2^{\omega}=\omega_{1}$, if they are consistent at all. This is because the failure of the principles of R. Jensen like in [De] or [Ve2] implies the consistency of the existence of an inaccessible cardinal. On the other hand, the usefulness of large cardinals in proofs of the consistency of the existence of small nice substructures of big structures is well known.

$\left({ }^{1}\right)$ By saying that an assertion is consistent we mean that if the usual axiomatization of mathematics, i.e., the axioms of ZFC are consistent, then the theory obtained by adding the assertion to ZFC is consistent as well. This is equivalent to the statement that the negation of the assertion cannot be proved from ZFC, unless ZFC has contradictions. See $[\mathrm{Ku}]$ for more explanations. 
The following versions of Johnson and Lindenstrauss's question emerge in the context of the results of $[\mathrm{Ko}]$ and the results of this paper:

1. Is there a compact totally disconnected Hausdorff space $K$ of arbitrarily large weight without infinite retracts of weight continuum (or any weight smaller than the weight of $K)$ ?

2. Is it consistent that every Banach space (of the form $C(K)$ ) has $\mathcal{C}\left(\omega, 2^{\omega}\right)$ ?

3. Is it true that in Gödel's constructible universe (where GCH holds) there are compact Hausdorff spaces $K$ of weight $\omega_{n}$ for $n>2$ such that $C(K)$ has no complemented subspaces of weight less than $\omega_{n}$ ?

4. Are there (consistently) indecomposable Banach spaces (of the form $C(K))$ of arbitrarily large density (bigger than the continuum)?

Note that our $K$ cannot have any infinite retracts of weight less than or equal to the continuum and that hereditarily indecomposable spaces have density less than or equal to the continuum (see [PY, Prop. 3.2]).

Complete understanding of the paper requires familiarity with the method of forcing within Chapter 7 of $[\mathrm{Ku}]$. However, forcing is just used to do the final trick. We have chosen this heuristically more complex approach since the other, via the principles like in [Ve1], seems much more technically complex.

As in $[\mathrm{Ko}]$, one can construct a Boolean algebra $\mathcal{A}$ as the union of an increasing sequence $\left(\mathcal{A}_{\alpha}\right)_{\alpha<2^{\omega}}$ of its subalgebras. If $K$ denotes its Stone space, then $C(K)$, especially under $\mathrm{CH}$, has remarkable properties, in particular every projection in $C(K)$ is a restriction to a clopen set plus a weakly compact operator. This implies that infinite-dimensional complemented subspaces of $C(K)$ have densities equal to weights of clopen sets. Here, we construct a Boolean algebra $\mathcal{A}$ with similar properties where the weights of clopen sets are bigger than the continuum, i.e., the densities of infinite-dimensional complemented subspaces are bigger than the continuum. Of course the algebra has to have cardinality bigger than the continuum, but constructing it as the union of an increasing sequence $(\mathcal{A})_{\alpha<\kappa}$ of its subalgebras for $\kappa>2^{\omega}$ is not the way we choose, since our techniques of [Ko] similar to those of [Fe], $[\mathrm{Ha}]$ or $[\mathrm{Ta}]$ do not work for a subalgebra of cardinality continuum. Instead we construct the algebra as the union of a directed system $\left(\mathcal{A}_{p}\right)_{p \in G}$, where $G$ is a partial order isomorphic to a certain family of countable subsets of $\kappa>2^{\omega}$ with the inclusion as the order. That is, we replace the countable ordinals (in the case of $2^{\omega}=\omega_{1}$ ) which are also countable sets of ordinals (less than the ordinal) by general countable sets of ordinals of a cardinal bigger than $2^{\omega}$. This allows us to construct a big structure (of size bigger than $2^{\omega}$ ) without going through uncountable intermediate stages. 
The construction has two stages. In the first (Section 2) we construct a partial order of countable algebras $\left(\mathcal{A}_{p}\right)_{p \in P}$ with some associated objects; not all algebras of this order are compatible, however some situations when they are compatible are indicated (Lemma 3). Section 2 also contains some facts about this partial order needed later. Then (Section 3) we use the method of forcing to obtain a $G \subseteq P$ which gives rise to a directed system of compatible algebras which can be amalgamated into one Boolean algebra with the required properties. Section 4 contains topological and analytic consequences of the construction. Thus, the understanding of forcing is needed just for verifying the short Section 3, however an extra effort is made to make the statements of this section understandable to mathematicians unfamiliar with forcing.

The notation is intended to be standard, some doubtful points are $f \mid A$ for the restriction of $f$ to $A$ and $f[A]$ for the image of $A$ under $f$, and $N$ for the set of natural numbers.

We will consider a classical chain of inter-related structures: a Boolean algebra $\mathcal{A}$, its Stone space $K$, the Banach space $C(K)$ of continuous functions on $K$ with the supremum norm, its dual, and the Banach space $M(K)$ of Radon measures on $K$ with the variation norm. Boolean algebras will be denoted by $\mathcal{A}, \mathcal{B}$, etc. The Boolean algebraic operations will be denoted $\vee$, $\wedge,-$, the complement by $A^{-1}$, also $A^{1}=A$; the minimal and the maximal elements will be denoted by 0 and 1 respectively, $\leq$ denotes the Boolean order. Most of the time the Boolean algebras we consider are subalgebras of the completion of a free algebra, so they are not fields of sets, but we will say that a family of their elements is pairwise disjoint when the meet of any two elements is 0 of the algebra. The subalgebra generated by a set $X$ is denoted by $\langle X\rangle$.

The Stone space $K$ of a Boolean algebra $\mathcal{A}$ consists of all ultrafilters and its basis consists of sets of the form

$$
[A]:=\{u \in K: A \in u\} \quad \text { for } A \in \mathcal{A} .
$$

Thus $x \in[A]$ is equivalent to $A \in x$. Note that we always have identities like $[A] \cup[B]=[A \vee B]$, but in general $\bigcup_{n \in N}\left[A_{n}\right] \neq\left[\bigvee_{n \in N} A_{n}\right]$ since the latter set is equal to $\overline{\bigcup_{n \in N}\left[A_{n}\right]}$ if the supremum exists, which will be used quite often. Books like [Si] can be consulted regarding Boolean algebras and the Stone duality.

The terminology concerning Banach spaces of continuous functions and linear operators follows [Se], [DS] and [Di]. Recall that a Radon measure on $K$ is a signed, Borel, scalar-valued, countably additive and regular measure. If $K$ is the Stone space of a Boolean algebra $\mathcal{A}$, then there is a unique Radon measure which extends a finitely additive bounded measure on the algebra $\mathcal{A}$; the latter is called a bounded measure on the algebra $\mathcal{A}$. The 
variation of a Radon measure $\mu$ is $|\mu|(K)$, where $|\mu(B)|$ for a Borel set $B \subseteq K$ is defined by

$|\mu|(B)=\sup \left\{\sum_{A \in \mathcal{F}}|\mu(A)|: \mathcal{F}\right.$ is a pairwise disjoint

finite family of Borel subsets of $B\}$.

It is well known that Radon measures on compact spaces have finite variation, that $|\mu|$ is a Radon measure assuming nonnegative values (and hence monotone) and that $|\mu|$ is the norm of a continuous linear functional on $C(K)$ given by $\int f d \mu$ for all $f \in C(K)$ (see [Se]). The regularity condition for a signed measure is that for any Borel $A \subseteq K$ and for any $\varepsilon>0$ there are compact $F \subseteq A$ and open $U \supseteq A$ such that $|\mu|(U-F)<\varepsilon$.

The Banach space of Radon measures with the variation norm will be denoted by $M(K)$. The terminology concerning partial orders is taken from forcing theory and follows $[\mathrm{Ku}]$; another classical textbook is [Je]. In particular, incompatible conditions are two elements $p, q$ of a partial order such that there is no $r \leq p, q$.

2. The partial order of countable approximations. Throughout this paper we are assuming the continuum hypothesis $\mathrm{CH}$. We may also assume that $2^{\omega_{1}}=\kappa$ for a previously chosen cardinal $\kappa$ of cofinality bigger than $\omega_{1}$. The consistency of such an assumption was proved by P. Cohen (see $[\mathrm{Ku}]$ ). For convenience, we will be assuming that $\kappa$ is a regular cardinal. We will be using a fact in ZFC, due to F. Hausdorff $([\mathrm{H}])$, that for any cardinal $\lambda$ there is a family of $2^{\lambda}$ independent sets, i.e., we need a family $\left(F_{\xi}: \xi<\kappa\right)$ of subsets of $\omega_{1}$ such that

$$
F_{\xi_{1}}^{\varepsilon_{1}} \cap \cdots \cap F_{\xi_{n}}^{\varepsilon_{n}} \neq \emptyset
$$

for any $\xi_{1}<\cdots<\xi_{n}<\kappa$ and $\varepsilon_{i} \in\{1,-1\}$ and $1 \leq i \leq n$. In particular we will be using the free Boolean algebra generated by an independent set of generators of cardinality $\kappa$ (which is the algebra of clopen sets of $2^{\kappa}$ with the Tikhonov topology). By the above facts we may conclude that (under our assumption) the Stone space of such an algebra has a dense set of ultrafilters of cardinality $\omega_{1}$. We will be working with the algebra $\mathcal{A}$, the completion of the free Boolean algebra with $\kappa$ generators $\left(F_{\xi}: \xi<\kappa\right)$, which by the above has a dense set of cardinality $\omega_{1}$ in its Stone space whose points (ultrafilters) will be denoted by $x_{\xi}$ for $\xi<\omega_{1}$. However, we should no longer use the interpretation of $F_{\xi}$ 's as subsets of $\omega_{1}$, because the infinitary operations in $\mathcal{A}$ do not coincide with the infinitary intersections or unions in $\wp\left(\omega_{1}\right)$. Two more facts about $\mathcal{A}$ which will be used are that $\mathcal{A}$ does not have a countable dense set, since $2^{\omega}<\kappa$, nor an uncountable set of pairwise disjoint elements; both facts follow from the topological interpretation of the 
free algebra as the algebra of clopen sets on $2^{\kappa}$. This implies, in particular, that each element of $\mathcal{A}$ is the supremum of a countable family of Boolean combinations like in $(*)$.

We define a partial order $P$ whose elements will be called conditions and will be of the form $p=\left(\mathcal{A}_{p}, \mathcal{P}_{p}\right)$, where

(a) $\mathcal{A}_{p}$ is a countable subalgebra of $\mathcal{A}$,

(b) $\mathcal{P}_{p}$ is a countable set of pairs of the form $(L, R)$, where $L$ and $R$ are disjoint countable subsets of $\omega_{1}$,

(c) for every $(L, R) \in \mathcal{P}_{p}$ the set $\left\{x_{\xi} \cap \mathcal{A}_{p}: \xi \in L \cup R\right\}$ is relatively discrete in the Stone space of $\mathcal{A}_{p}$,

$$
\overline{\left\{x_{\xi} \cap \mathcal{A}_{p}: \xi \in L\right\}} \cap \overline{\left\{x_{\xi} \cap \mathcal{A}_{p}: \xi \in R\right\}} \neq \emptyset
$$

for every $(L, R) \in \mathcal{P}_{p}$, where the closures are taken in the Stone space of $\mathcal{A}_{p}$.

The elements of $\mathcal{P}_{p}$ will be called promises. One can express the property of being a condition without referring to the Stone space of $\mathcal{A}_{p}$.

Lemma 1. $\left(\mathcal{A}_{p}, \mathcal{P}_{p}\right)$ is a condition of $P$ if and only if $(\mathrm{a}),(\mathrm{b})$ are satisfied and

$\left(\mathrm{c}^{\prime}\right)$ for every $(L, R) \in \mathcal{P}_{p}$ there is an antichain $\left(A_{\xi}: \xi \in L \cup R\right)$ in $\mathcal{A}_{p}$ such that $A_{\xi} \in x_{\eta}$ if and only if $\xi=\eta$,

$\left(\mathrm{d}^{\prime}\right)$ there is no element $A$ of $\mathcal{A}_{p}$ such that $A \in x_{\xi}$ if $\xi \in L$ and $A \notin x_{\xi}$ if $\xi \in R$.

An element $A$ like in $\left(\mathrm{d}^{\prime}\right)$ is said to separate $L$ and $R$.

Proof. This follows from the fact that Stone spaces are compact and totally disconnected.

The order is defined by $p \leq q$ if and only if $\mathcal{A}_{p} \supseteq \mathcal{A}_{q}$ and $\mathcal{P}_{p} \supseteq \mathcal{P}_{q}$. Note that bigger in the combinatorial sense means smaller in the order sense, but we will call it stronger, following an established convention in forcing which has a good justification (see $[\mathrm{Ku}]$ ).

Lemma 2. $P$ is $\sigma$-closed, i.e., any decreasing countable sequence of conditions of $P$ has a lower bound in $P$.

Proof. Assume that $p_{n}$ 's are in $P$ and $p_{n+1} \leq p_{n}$ for all $n \in N$. Define $p=\left(\mathcal{A}_{p}, \mathcal{P}_{p}\right)$ by $\mathcal{A}_{p}=\bigcup_{n \in N} \mathcal{A}_{p_{n}}$ and $\mathcal{P}_{p}=\bigcup_{n \in N} \mathcal{P}_{p_{n}}$. Clearly $\mathcal{A}_{p}$ is a countable subalgebra of $\mathcal{A}$ bigger than all subalgebras $\mathcal{A}_{p_{n}}$. By Lemma 1 the separation of any $(L, R) \in \mathcal{P}_{p_{n}}$ would be witnessed by some element of $\mathcal{A}_{p_{m}}$ for some $m$, which would contradict the fact that $\left(\mathcal{A}_{p_{k}}, \mathcal{P}_{p_{k}}\right)$ is a condition of $P$ for $k=\max (n, m)$.

Lemma 3. $P$ satisfies the $\omega_{2}$-chain condition, i.e., there is no pairwise incompatible set of conditions of $P$ of cardinality $\omega_{2}$. 
Proof. Suppose that $\left(p_{\xi}: \xi<\omega_{2}\right)$ is a sequence of distinct elements of $P$. By $\mathrm{CH}$ we may assume without loss of generality that $\mathcal{P}_{p_{\xi}}=\mathcal{P}$ for some $\mathcal{P}$ and all $\xi<\omega_{2}$ and that $L \cup R \subseteq \alpha$ for some $\alpha<\omega_{1}$ for all $(L, R) \in \mathcal{P}$. Further thinning out the original sequence and using $\mathrm{CH}$ we may assume without loss of generality that for any $\xi<\xi^{\prime}<\omega_{2}$ for a set $X \subseteq \alpha$ the existence of an $A \in \mathcal{A}_{p_{\xi}}$ such that $A \in x_{\eta}$ if and only if $\eta \in X$ is equivalent to the existence of an $A^{\prime} \in \mathcal{A}_{p_{\xi^{\prime}}}$ such that $A^{\prime} \in x_{\eta}$ if and only if $\eta \in X$. Now let us see that $p$ is a stronger condition than two $p_{\xi}$ and $p_{\xi^{\prime}}$, where $\mathcal{A}_{p}$ is the Boolean algebra generated by $\mathcal{A}_{p_{\xi}}$ and $\mathcal{A}_{p_{\xi^{\prime}}}$, and $\mathcal{P}_{p}=\mathcal{P}$ is as above.

For this we need to check condition $\left(\mathrm{d}^{\prime}\right)$. But all elements of $\mathcal{A}_{p}$ are of the form $\bigvee_{i \leq n}\left(A_{i} \wedge B_{i}\right)$, where $A_{i} \in \mathcal{A}_{p_{\xi}}$ and $B_{i} \in \mathcal{A}_{p_{\xi^{\prime}}}$, so by the above obtained properties of the sequence of our conditions we have

$$
\begin{aligned}
\left\{\eta \in \alpha: \bigvee_{i \leq n}\left(A_{i} \wedge B_{i}\right) \in x_{\eta}\right\} & =\left\{\eta \in \alpha: \bigvee_{i \leq n}\left(A_{i} \wedge A_{i}^{\prime}\right) \in x_{\eta}\right\} \\
& =\left\{\eta \in \alpha: \bigvee_{i \leq n}\left(B_{i}^{\prime} \wedge B_{i}\right) \in x_{\eta}\right\}
\end{aligned}
$$

for some $A_{i}^{\prime} \in \mathcal{A}_{p_{\xi}}$ and $B_{i}^{\prime} \in \mathcal{A}_{p_{\xi^{\prime}}}$. So, among the above sets there cannot appear a set which separates $L$ and $R$ for some $(L, R)$ from $\mathcal{P}_{p}=\mathcal{P}_{p_{\xi}}=\mathcal{P}_{p_{\xi}^{\prime}}$. This shows that the original sequence has two (actually $\omega_{2}$ ) compatible conditions, completing the proof of the lemma.

The remaining lemmas of this section are "density lemmas", that is, they show that for any condition $p \in P$, there is a stronger condition $q \leq p$ with some interesting properties. $q$ is going to "force" the final construction to have the interesting property. Recall that a subset $D$ of a partial order $P$, in forcing theory, is called dense if and only if for every $p \in P$ there is $d \in D$ satisfying $d \leq p$.

Lemma 4. Suppose that $S \subseteq \kappa$ with $|S|<\kappa, p \in P$ and $0 \neq A \in \mathcal{A}_{p}$. Then there is $q \leq p$ and $0 \neq B \in \mathcal{A}_{q}$ with $B \leq A$ such that $B=F_{\xi_{1}}^{\varepsilon_{1}} \wedge$ $\cdots \wedge F_{\xi_{n}}^{\varepsilon_{n}}$ for some $\xi_{1}<\cdots<\xi_{n}<\kappa$ satisfying $\left\{\xi_{1}, \ldots, \xi_{n}\right\} \nsubseteq S$, for some $\varepsilon_{i} \in\{1,-1\}, 1 \leq i \leq n$.

Proof. $[A]$ is not separable, hence there is a $B \leq A$ such that $[B]$ is disjoint from the closure of $\left\{x_{\xi}: \xi \in L \cup R,(L, R) \in \mathcal{P}_{p}\right\}$. By taking a smaller $B$ we can assume that it is a Boolean combination as in the statement of the lemma.

It is clear that $q=\left(\left\langle\mathcal{A}_{p} \cup\{B\}\right\rangle, \mathcal{P}_{p}\right)$ is a condition of $P$ since all the promises from $\mathcal{P}_{p}$ are preserved by the choice of $B$.

Lemma 5. Suppose that $\mathcal{B}$ is a countable subalgebra of $\mathcal{A}$ and that both $\left(x_{\xi_{k}} \cap \mathcal{B}\right)_{k \in N}$ and $\left(x_{\eta_{k}} \cap \mathcal{B}\right)_{k \in N}$ are sequences of distinct elements which converge to one point in the Stone space of $\mathcal{B}$. Suppose that $C$ is an element 
of $\mathcal{A}$ such that there are infinitely many $k$ 's satisfying $A_{k} \in\left(x_{\xi_{k}} \cap \mathcal{B}\right)$ and $A_{k} \leq C$ as well as there are infinitely many $k$ 's satisfying $B_{k} \in\left(x_{\eta_{k}} \cap \mathcal{B}\right)$ and $B_{k} \leq C$. Then in the Stone space of $\langle\mathcal{B} \cup\{C\}\rangle$ we have

$$
\overline{\left\{x_{\xi_{k}} \cap\langle\mathcal{B} \cup\{C\}\rangle: k \in N\right\}} \cap \overline{\left\{x_{\eta_{k}} \cap\langle\mathcal{B} \cup\{C\}\rangle: k \in N\right\}} \neq \emptyset .
$$

Proof. As in Lemma 1, it is enough to see that there is no element in $\langle\mathcal{B} \cup\{C\}\rangle$ which separates $\left\{x_{\xi_{k}} \cap\langle\mathcal{B} \cup\{C\}\rangle: k \in N\right\}$ from $\left\{x_{\eta_{k}} \cap\langle\mathcal{B} \cup\{C\}\rangle\right.$ : $k \in N\}$. But any element of $\langle\mathcal{B} \cup\{C\}\rangle$ is of the form $G=(D \wedge C) \vee(E-C) \vee F$, where $D, E, F$ are pairwise disjoint elements of $\mathcal{B}$, i.e., only one of them can belong to the limit point of the sequences $\left(x_{\xi_{k}} \cap \mathcal{B}\right)_{k \in N}$ and $\left(x_{\eta_{k}} \cap \mathcal{B}\right)_{k \in N}$, i.e., either $G$ does not belong to all but finitely many terms of the sequences or one and only one of the elements $D, E, F$ belongs to all but finitely many terms of the sequences.

Considering the three cases and the fact that $C$ belongs to infinitely many points $x_{\xi_{k}} \cap\langle\mathcal{B} \cup\{C\}\rangle$ as well as to infinitely many points $x_{\eta_{k}} \cap\langle\mathcal{B} \cup\{C\}\rangle$ we conclude that $G$ cannot separate the sequences, which completes the proof of the lemma.

Note that the algebras $\langle\mathcal{B} \cup\{C\}\rangle$ and $\langle\mathcal{B} \cup\{1-C\}\rangle$ are the same, so the condition $A_{k} \leq C$ for infinitely many $k$ 's and $B_{k} \leq C$ for infinitely many $k$ 's may be replaced in Lemma 5 by the condition that requires $A_{k} \wedge C=0$ for infinitely many $k$ 's and $B_{k} \wedge C=0$ for infinitely many $k$ 's.

Lemma 6. Suppose $p \in P,\left\{x_{\alpha_{n}} \cap \mathcal{A}_{p}: n \in N\right\}$ is relatively discrete in the Stone space of $\mathcal{A}_{p}$ and $A_{n} \in \mathcal{A}_{p}$ are pairwise disjoint and such that $A_{m} \notin x_{\alpha_{n}}$ for all $n, m \in N$ and there are at most finitely many $\xi$ 's in $\left\{L \cup R:(L, R) \in \mathcal{P}_{p}\right\} \cup\left\{\alpha_{n}: n \in N\right\}$ satisfying

$$
x_{\xi} \cap \mathcal{A}_{p} \in \overline{\bigcup_{n \in N}\left[A_{n}\right]}-\bigcup_{n \in N}\left[A_{n}\right],
$$

where the closures and unions are taken in the Stone space of $\mathcal{A}_{p}$. Then there is an infinite $M \subseteq N$ such that for every infinite $M^{\prime} \subseteq M$ and for any two pairwise disjoint infinite $L, R \subseteq\left\{\alpha_{n}: n \in N\right\}$ satisfying

$$
\overline{\left\{x_{\alpha_{n}} \cap \mathcal{A}_{p}: \alpha_{n} \in L\right\}} \cap \overline{\left\{x_{\alpha_{n}} \cap \mathcal{A}_{p}: \alpha_{n} \in R\right\}} \neq \emptyset,
$$

an element

$$
q_{M^{\prime}}=\left(\left\langle\mathcal{A}_{p} \cup\left\{\bigvee_{m \in M^{\prime}} A_{m}\right\}\right\rangle, \mathcal{P}_{p} \cup\{L, R\}\right)
$$

is a condition of $P$.

Proof. The only problem to worry about is keeping the promises from $\mathcal{P}_{p} \cup\{L, R\}$. As $\mathcal{A}_{p}$ is countable, the Stone space of $\mathcal{A}_{p}$ is metrizable, hence the condition (d) translates into the existence of points $x^{m}$ of the Stone space of $\mathcal{A}_{p}$ and pairs of convergent sequences of distinct elements $x_{k}^{m}, y_{k}^{m} \rightarrow x^{m}$, 
$u_{m}, v_{m} \rightarrow w$, where $x_{k}^{m} \in\left\{x_{\xi} \cap \mathcal{A}_{p}: \xi \in L_{m}\right\}, y_{k}^{m} \in\left\{x_{\xi} \cap \mathcal{A}_{p}: \xi \in R_{m}\right\}$, $\mathcal{P}_{p}=\left\{\left(L_{m}, R_{m}\right): m \in N\right\},\left\{u_{m}: m \in N\right\} \subseteq\left\{x_{\xi}: \xi \in L\right\}$ and $\left\{v_{m}: m \in N\right\}$ $\subseteq\left\{x_{\xi}: \xi \in R\right\}$.

Since $\left\{x_{\alpha_{n}} \cap \mathcal{A}_{p}: n \in N\right\}$ is relatively discrete,

$$
\overline{\left\{x_{\alpha_{n}} \cap \mathcal{A}_{p}: \alpha_{n} \in L-F\right\}} \cap \overline{\left\{x_{\alpha_{n}} \cap \mathcal{A}_{p}: \alpha_{n} \in R-F\right\}} \neq \emptyset
$$

holds for any finite $F$, and so without loss of generality we may assume that no $x_{\alpha_{n}}$ belongs to

$$
\overline{\bigcup_{n \in N}\left[A_{n}\right]}-\bigcup_{n \in N}\left[A_{n}\right]
$$

By the fact that $A_{m} \notin x_{\alpha_{n}}$ for all $n, m \in N$, this means that there are $A_{n}^{\prime} \in \mathcal{A}_{p} \cap x_{\alpha_{n}}$ satisfying

$$
A_{n}^{\prime} \wedge \bigvee_{m \in N} A_{m}=0
$$

which by the observation made after Lemma 5 and by Lemma 5 means that the promise $(L, R)$ is always kept. Thus we will only deal with the remaining promises.

If $x_{m} \notin \overline{\bigcup_{n \in N}\left[A_{n}\right]}-\bigcup_{n \in N}\left[A_{n}\right]$, then there is $A \in \mathcal{A}_{p}$ such that $A$ belongs to eventually all ultrafilters $x_{k}^{m}$ and $y_{k}^{m}$ and $A \leq \bigvee_{m \in M^{\prime}} A_{m}$ or $A \wedge \bigvee_{m \in M^{\prime}} A_{m}=0$ for any $M^{\prime} \subseteq N$. This means, by Lemma 5 , that there is no element of the algebra $\left\langle\mathcal{A}_{p} \cup\left\{\bigvee_{m \in M^{\prime}} A_{m}\right\}\right\rangle$ which separates $L_{m}$ and $R_{m}$ for such an $m$.

Thus, by the above and the assumption we may assume without loss of generality that

$$
x^{m} \in \overline{\bigcup_{n \in N}\left[A_{n}\right]}-\bigcup_{n \in N}\left[A_{n}\right],
$$

which implies that each of our convergent sequences may have at most finitely many points in any fixed set $\left[A_{n}\right]$ and

$$
x_{k}^{m}, y_{k}^{m} \notin \overline{\bigcup_{n \in N}\left[A_{n}\right]}-\bigcup_{n \in N}\left[A_{n}\right]
$$

for all $m, k \in N$. For $m \in N$ define

$$
\begin{aligned}
& T_{L}^{m}=\left\{n \in N: \exists k \in N, x_{k}^{m} \in\left[A_{n}\right]\right\}, \\
& T_{R}^{m}=\left\{n \in N: \exists k \in N, y_{k}^{m} \in\left[A_{n}\right]\right\} .
\end{aligned}
$$

Let $M$ be any infinite subset of $N$ whose complement intersects in an infinite set each infinite $T_{L}^{m}$ and each infinite $T_{R}^{m}$. It is easy to obtain such a set since there are only countably many $T_{L}^{m}$ 's and $T_{R}^{m}$ 's. Any infinite subset $M^{\prime}$ of $M$ has the same property. This means that for every $m$ there are infinitely many $k$ 's such that there is a $B_{k}^{m} \in x_{k}^{m}$ satisfying $B_{k}^{m} \leq 1-\bigvee_{m \in M^{\prime}} A_{m}$ and there are infinitely many $k$ 's such that there is a $C_{k}^{m} \in y_{k}^{m}$ satisfying 
$C_{k}^{m} \leq 1-\bigvee_{m \in M^{\prime}} A_{m}$. This means by Lemma 5 that the promises $\left(L_{m}, R_{m}\right)$ are preserved in $\mathcal{A}_{q_{M^{\prime}}}$, i.e., $q_{M^{\prime}}$ is a condition.

The hypothesis of the following lemma mentions bounded measures on a Boolean algebra. The statement of the lemma mentions the variation of the measures, which is usually defined for Radon measures. One needs to use the fact that there is a one-to-one correspondence between these concepts, i.e., we can extend the measure on the Boolean algebra to the unique Radon measure on the Borel sets and use the variation for this measure. However one can also define variation for a bounded measure (see [Se, 17.2.1]) on a Boolean algebra which by the regularity condition coincides with the variation of its Radon extension.

Lemma 7. Suppose that $p \in P$ and that we are given

(a) a sequence of pairwise disjoint elements $\left(A_{n}: n \in N\right)$ of $\mathcal{A}_{p}$,

(b) a sequence $\left(\alpha_{n}: n \in N\right) \subseteq \omega_{1}$ such that $A_{m} \notin x_{\alpha_{n}}$ for all $n, m \in N$, and $\left(x_{\alpha_{n}} \cap \mathcal{A}_{p}\right)_{n \in N}$ is relatively discrete,

(c) an $\varepsilon>0$,

(d) a sequence $\left(\mu_{n}: n \in N\right)$ of bounded measures on the algebra $\mathcal{A}_{p}$ such that $\left|\mu_{n}\left(A_{n}\right)\right|>\varepsilon$ for each $n \in N$.

Then there are $q \leq p, \delta>0$, infinite $b \subseteq a \subseteq N$ and elements $A_{n}^{\prime} \leq A_{n}$ of $\mathcal{A}_{p}$ for $n \in$ a such that

(e) the supremum $\bigvee\left\{A_{n}^{\prime}: n \in b\right\}$ belongs to $\mathcal{A}_{q}$,

(f) $\left|\mu_{n}\left(A_{n}^{\prime}\right)\right|>\delta$ and $\sum\left\{\left|\mu_{n}\right|\left(A_{m}^{\prime}\right): m \neq n, m \in a\right\}<\delta / 3$ for all $n \in a$,

(g) $\left(\left\{\alpha_{n}: n \in b\right\},\left\{\alpha_{n}: n \in a-b\right\}\right) \in \mathcal{P}_{q}$.

Proof. Let $\left\{\xi_{n}: n \in N\right\}=\bigcup\left\{L \cup R:(L, R) \in \mathcal{P}_{p}\right\} \cup\left\{\alpha_{n}: n \in N\right\}$. First we will be working in the Stone space of $\mathcal{A}_{p}$. We need to thin out the $A_{n}$ 's to $A_{n}^{\prime}$ 's for $n$ in some infinite subset $N_{1} \subseteq N$ so that

(1) $A_{n}^{\prime} \subseteq A_{n},\left|\mu_{n}\left(A_{n}^{\prime}\right)\right|>\delta^{\prime}$ for some $\delta^{\prime}>0$ and $n \in N_{1}$ and either

(2) $\overline{\bigcup_{n \in N_{1}} A_{n}^{\prime}}-\bigcup_{n \in N_{1}} A_{n}^{\prime}$ is one point in the Stone space of $\mathcal{A}_{p}$, or

(3) none of the points $x_{\xi_{n}} \cap \mathcal{A}_{p}$ for $n \in \mathbb{N}$ is in $\overline{\bigcup_{n \in N_{1}} A_{n}^{\prime}}-\bigcup_{n \in N_{1}} A_{n}^{\prime}$.

In either case (2) or (3) the hypothesis of Lemma 6 will be satisfied.

Case 1: There is $\delta^{\prime}>0$ and a point $x$ in the Stone space of $\mathcal{A}_{p}$ such that for each clopen neighbourhood $A$ of $x$ and for every $m \in N$ there is $k>m$ such that $\left|\mu_{k}\right|\left(A_{k} \wedge A\right)>\delta^{\prime}$. As $A_{n}$ 's are pairwise disjoint we may assume without loss of generality that $x$ does not belong to any of them. Since the neighbourhood basis at $x$ is countable we can construct by induction a pairwise disjoint sequence $\left(A_{n}^{\prime}\right)_{n \in N_{1}}$ of clopen sets of the form $A_{k} \wedge A$ as above which do not contain $x$, such that any neighbourhood of $x$ includes almost all sets $A_{n}^{\prime}$, i.e., $(2)$ is satisfied and $A_{n}^{\prime} \subseteq A_{n}$ and $\left|\mu_{n}\right|\left(A_{n}^{\prime}\right)>\delta^{\prime}$ for 
$n \in N_{1}$. Applying [Se, 17.2.2] and the regularity of the measures we may assume without loss of generality that $\left|\mu_{n}\left(A_{n}^{\prime}\right)\right|>\delta^{\prime}$ for $n \in M$.

CASE 2: Case 1 does not hold. For every $n \in N$ and for every $\delta^{\prime}>0$ there is an $m\left(n, \delta^{\prime}\right) \in N$ and $A\left(n, \delta^{\prime}\right)$ in $x_{\xi_{n}} \cap \mathcal{A}_{p}$ such that

$$
\left|\mu_{k}\right|\left(A_{k} \wedge A\left(n, \delta^{\prime}\right)\right)<\delta^{\prime}
$$

for $k>m\left(n, \delta^{\prime}\right)$. Thus, one can choose by induction a strictly increasing sequence of integers $\left(k_{n}\right)_{n \in N}$ such that $k_{n}>m\left(j, \varepsilon / 2^{j+2}\right)$ for all $j<n$. Consider

$$
A_{k_{n}}^{\prime}=A_{k_{n}}-\bigvee\left\{A\left(j, \varepsilon / 2^{j+2}\right): j<n\right\}
$$

By $(*)$ we have $\left|\mu_{k_{n}}\right|\left(A_{k_{n}} \wedge A\left(j, \varepsilon / 2^{j+2}\right)\right)<\varepsilon / 2^{j+2}$ for $j<n$ and so

$$
\left|\mu_{k_{n}}\right|\left(A_{k_{n}}^{\prime}\right)>\varepsilon / 2=\delta \text {. }
$$

Now, note that $A\left(n, \varepsilon / 2^{n+2}\right)$ is disjoint from $A_{k_{i}}^{\prime}$ for $i>n$ and hence $x_{\xi_{n}} \cap \mathcal{A}_{p}$ 's do not belong to the closure of the set as in (3), where $N_{1}=\left\{k_{n}\right.$ : $n \in N\}$ as required in (3).

Thus in both cases we have (1) and either (2) or (3). Now we apply Rosenthal's lemma (see [Ro, Lemma 1.1]; for the stronger version that we are using see [Di, p. 82]) to find an infinite $N_{2} \subseteq N_{1}$ such that

$$
\sum\left\{\left|\mu_{n}\right|\left(A_{m}^{\prime}\right): n \neq m, m \in N_{2}\right\}<\delta / 3 \text {. }
$$

Finally, note that (2) or (3) imply that the hypothesis of Lemma 6 is satisfied. Let $M$ be as in Lemma 6 .

For every infinite $a \subseteq M$ there is an infinite $b \subseteq a$ such that the set $\overline{\left\{x_{\alpha_{n}} \cap \mathcal{A}_{p}: n \in b\right\}}$ intersects $\overline{\left\{x_{\alpha_{n}} \cap \mathcal{A}_{p}: n \in a-b\right\}}$, where the closures are taken in the Stone space of $\mathcal{A}_{p}$. This follows from the fact that the topological weight of the Stone space of $\mathcal{A}_{p}$ is countable, and in a compact space separations of closed sets can be done by finitely many basic open sets. Define $L=\left\{\alpha_{n}: n \in b\right\}$ and $R=\left\{\alpha_{n}: n \in a-b\right\}$ for such $a$ and $b$. By Lemma 6 ,

$$
q=\left(\left\langle\mathcal{A}_{p} \cup\left\{\bigvee_{m \in b} A_{m}^{\prime}\right\}\right\rangle, \mathcal{P}_{p} \cup\{(L, R)\}\right)
$$

is a condition of $P$, by the construction (e), (g) hold, and (f) holds by (**) and $(* * *)$, which completes the proof of the lemma.

Lemma 8. Let $p \in P$ and $A_{n}$ and $B_{n}$ be elements of $\mathcal{A}_{p}$ for $n \in N$ such that

(a) $A_{n} \cap B_{m}=\emptyset$ for $n, m \in N$,

(b) $\overline{\bigcup\left\{\left[A_{n}\right]: n \in N\right\}} \cap \overline{\bigcup\left\{\left[B_{n}\right]: n \in N\right\}} \neq \emptyset$.

Then there is $q \leq p$ with $\left(L_{1}, R_{1}\right),\left(L_{2}, R_{2}\right) \in \mathcal{P}_{q}$ and an $A \in \mathcal{A}_{q}$ such that

(c) $\forall \alpha \in L_{1} \cup L_{2} \exists n \in N \quad A_{n} \in x_{\alpha} \cap \mathcal{A}_{p}$, 
(d) $\forall \alpha \in R_{1} \cup R_{2} \exists n \in N \quad B_{n} \in x_{\alpha} \cap \mathcal{A}_{p}$,

(e) $\forall \alpha \in L_{1} \cup R_{1} \forall \beta \in L_{2} \cup R_{2} \quad A \in x_{\alpha}, A \notin x_{\beta}$.

Proof. Since the Stone space of $\mathcal{A}_{p}$ is metrizable, there are distinct $\xi_{m}$ and $\eta_{m}$ in $\omega_{1}$ such that both $\left(x_{\xi_{m}} \cap \mathcal{A}_{p}\right)_{m \in N}$ and $\left(x_{\eta_{m}} \cap \mathcal{A}_{p}\right)_{m \in N}$ converge to a single point $x$ of the Stone space of $\mathcal{A}_{p}$ and for each $m \in N$ there are $n_{m}, n_{m}^{\prime} \in N$ such that $A_{n_{m}} \in x_{\xi_{m}}$ and $B_{n_{m}^{\prime}} \in x_{\eta_{m}}$.

Since the neighbourhood basis at $x$ is countable, one can find pairwise disjoint $A_{m}^{\prime}$ and $B_{m}^{\prime}$ such that $A_{m}^{\prime} \in x_{\xi_{m}}$ and $B_{m}^{\prime} \in x_{\eta_{m}}$ and $A_{m}^{\prime} \subseteq A_{n_{m}}$ and $B_{m}^{\prime} \subseteq B_{n_{m}^{\prime}}$ and every neighbourhood of $x$ contains all but finitely many $A_{m}^{\prime}$ and $B_{m}^{\prime}$. The latter implies that

$$
\overline{\bigcup_{m \in N}\left[A_{m}^{\prime} \vee B_{m}^{\prime}\right]}-\bigcup_{m \in N}\left[A_{m}^{\prime} \vee B_{m}^{\prime}\right]
$$

is just the single point $x$. Hence, by Lemma 6 , there is an infinite $M^{\prime} \subseteq 2 N$ such that

$$
q_{M^{\prime}}=\left(\left\langle\mathcal{A}_{p} \cup\left\{\bigvee_{m \in M^{\prime}}\left(A_{m}^{\prime} \vee B_{m}^{\prime}\right)\right\}\right\rangle, \mathcal{P}_{p}\right)
$$

is a condition. Note that by Lemma 5 ,

$$
q=\left(\left\langle\mathcal{A}_{p} \cup\left\{\bigvee_{m \in M^{\prime}}\left(A_{m}^{\prime} \vee B_{m}^{\prime}\right)\right\}\right\rangle, \mathcal{P}_{p} \cup\left\{\left(L_{1}, R_{1}\right),\left(L_{2}, R_{2}\right)\right\}\right)
$$

is a condition, where $L_{1}=\left\{\xi_{m}: m \in M^{\prime}\right\}, R_{1}=\left\{\eta_{m}: m \in M^{\prime}\right\}, L_{2}=$ $\left\{\xi_{m}: m \in N-2 N\right\}, R_{2}=\left\{\eta_{m}: m \in N-2 N\right\}$.

Now $\left.A=\bigvee_{m \in M^{\prime}}\left(A_{m}^{\prime} \vee B_{m}^{\prime}\right)\right\}$ satisfies the assertion of the lemma.

3. The generic construction. By Lemmas 2 and 3 , forcing with $P$ does not collapse cardinals and does not add any new countable subsets of the universe (see $[\mathrm{Ku}]$ ), i.e., the ground set-theoretic universe $V$ and the generic extension $V[G]$ have the same countable sets of $V$ and the same cardinals.

Let $G$ be a $P$-generic filter over a set-theoretic universe $V$. That is, for any two $p, p^{\prime} \in G$ we have $q \leq p, p^{\prime}$ for some $q \in G$, and $G \cap D \neq \emptyset$ for any dense set $D \subseteq P$ in the ground set-theoretic universe $V$. In the generic extension $V[G]$ we consider the Boolean algebra $\mathcal{A}^{*}=\bigcup\left\{\mathcal{A}_{p}: p \in G\right\}$ generated in $\mathcal{A}$. The fact that for any finite collection of elements of a generic filter $G$ there is a stronger condition in $G$ implies that $\mathcal{A}^{*}$ is a Boolean algebra. Note that $\mathcal{A}$ is still a completion of the free Boolean algebra with $\kappa$ generators, since it is a c.c.c. algebra and there are no new countable sets of $\mathcal{A}$ in $V[G]$.

Lemma 9. The algebra $\mathcal{A}$ has the following properties:

(A) Below any $0 \neq A \in \mathcal{A}^{*}$ there are $\kappa$ distinct elements of $\mathcal{A}$. 
(B) Given

(a) a sequence of pairwise disjoint elements $\left(A_{n}: n \in N\right)$ of $\mathcal{A}^{*}$,

(b) a sequence $\left(\alpha_{n}: n \in N\right) \subseteq \omega_{1}$ such that $A_{m} \notin x_{\alpha_{n}}$ for all $n, m \in N$, and $\left\{x_{\alpha_{n}}: n \in N\right\}$ is relatively discrete,

(c) an $\varepsilon>0$,

(d) a sequence $\left(\mu_{n}: n \in N\right)$ of bounded measures on the algebra $\mathcal{A}^{*}$ such that $\left|\mu_{n}\left(A_{n}\right)\right|>\varepsilon$ for each $n \in N$,

there are $\delta>0$, infinite $b \subseteq a \subseteq N$ and elements $A_{n}^{\prime} \leq A_{n}$ of $\mathcal{A}^{*}$ for $n \in$ a such that

(e) the supremum $A=\bigvee\left\{A_{n}^{\prime}: n \in b\right\}$ exists in $\mathcal{A}^{*}$,

(f) $\left|\mu_{n}\left(A_{n}^{\prime}\right)\right|>\delta$ and $\sum\left\{\left|\mu_{n}\right|\left(A_{m}^{\prime}\right): m \neq n, m \in a\right\}<\delta / 3$ for all $n \in a$,

(g) there is no $B$ in $\mathcal{A}^{*}$ such that $n \in b$ implies $B \in x_{\alpha_{n}}$ and $n \in a-b$ implies $B \notin x_{\alpha_{n}}$.

(C) If $U_{1}, U_{2}$ are two open subsets of the Stone space of $\mathcal{A}$, then $\bar{U}_{1} \cap \bar{U}_{2}=\emptyset$ or $\bar{U}_{1} \cap \bar{U}_{2}$ has at least two points.

Proof. Suppose that (A) is false; since $\mathcal{A}$ and so $\mathcal{A}^{*}$ are c.c.c., this implies that there is a condition $p \in P$ which forces that $0 \neq A \in \mathcal{A}^{*}$ and that the family of elements of $\mathcal{A}^{*}$ smaller than $A$ is included in the completion of the algebra generated by $\left\{\mathcal{F}_{\xi}: \xi \in \mathcal{S}\right\}$ for some $S \subseteq \kappa$ of cardinality less than $\kappa$. Since $P$ is $\omega_{2}$-c.c. and $\kappa$ is regular we may assume that $S$ is in the ground model.

Lemma 4 implies that there is a $q \leq p$ which forces that there is an element in $\mathcal{A}^{*}$ smaller than $A$ which is not in the completion of the algebra generated by $\left\{F_{\xi}: \xi \in S\right\}$, a contradiction.

To get (B) work in $V$ and fix $p \in P$. Let $\dot{A}_{n}, \dot{\mu}_{n}, \dot{\varepsilon}$ be $P$-names for the objects mentioned in items (a)-(d) of (B). We will produce $q \leq p$ which will force (e)-(g) of the lemma. Since $P$ forces that $\dot{A}_{n}$ 's are in $\dot{\mathcal{A}}^{*}$, there are $A_{n}$ 's and a strictly decreasing sequence $\left(p_{n}\right)_{n \in N}$ of conditions of $P$ such that $p_{n} \|-\dot{A}_{n}=\check{A}_{n}$. We may assume without loss of generality that $A_{n} \in \mathcal{A}_{p_{n}}$. By Lemma 2 there is $p$ stronger than all $p_{n}$ 's. Note that $A_{n}$ 's are in $\mathcal{A}_{p}$. We can also assume that the value of $\dot{\varepsilon}>0$ is decided as no reals are added by $P$. Using a procedure as above we may construct another decreasing sequence of $p_{n}$ 's such that for any $n \in N$ and $A \in \mathcal{A}_{p_{m}}$ there is a $k \in N$ such that the value of $\dot{\mu}_{n}(A)$ is decided by $p_{k}$. Now for $p$ such that $\mathcal{A}_{p}=\bigcup_{n \in N} \mathcal{A}_{p_{n}}$ there exist functions $\mu_{n}: \mathcal{A}_{p} \rightarrow \mathbb{R}$ such that

$$
\forall A \in \mathcal{A}_{p} \quad p \Vdash \check{\mu}_{n}(A)=\dot{\mu}_{n}(A) .
$$

This in particular implies that $\mu_{n}$ 's are measures on $\mathcal{A}_{p}$. Now Lemma 7 implies that there is $q \leq p$ which forces (e)-(g). 
To get (C), assume that $\bar{U}_{1} \cap \bar{U}_{2} \neq \emptyset$ and note that by the c.c.c. of $\mathcal{A}^{*}$ we have countable sequences $\left\{A_{n}: n \in N\right\}$ and $\left\{B_{n}: n \in N\right\}$ of elements of $\mathcal{A}^{*}$ such that $\left[A_{n}\right] \subseteq U_{1}$ and $\left[B_{n}\right] \subseteq U_{2}$ and $\overline{\bigcup\left\{\left[A_{n}\right]: n \in N\right\}} \cap \overline{\bigcup\left\{\left[B_{n}\right]: n \in N\right\}}$ $\neq \emptyset$. Using the procedure like in the proof of $(\mathrm{B})$ one can assume without loss of generality that there is a condition $p \in P$ and sequences $\left\{A_{n}: n \in N\right\}$ and $\left\{B_{n}: n \in N\right\}$ of elements of $\mathcal{A}_{p}$ with $\overline{\bigcup\left\{\left[A_{n}\right]: n \in N\right\}} \cap \overline{\bigcup\left\{\left[B_{n}\right]: n \in N\right\}} \neq \emptyset$ in the Stone space of $\mathcal{A}_{p}$ such that $p$ forces that $A_{n} \subseteq U_{1}$ and $B_{n} \subseteq U_{2}$. Lemma 8 implies that there is $q \leq p$ which forces that there are at least two points in $\bar{U}_{1} \cap \bar{U}_{2}$, one in $A$ and the other in the complement of $A$, where $A$ is as in Lemma 8.

4. Consequences of Boolean algebraic properties of $\mathcal{A}^{*}$. In this section $K$ stands for the Stone space of the algebra $\mathcal{A}^{*}$ constructed in the previous section. All the properties of the algebra used in this section are stated in Lemma 9. All the arguments of this section are minor modifications of the arguments from [Ko]. When possible we will provide the exact reference, otherwise we will repeat an analogous argument for the convenience of the reader.

Proposition 10. The Stone space $K$ of the algebra $\mathcal{A}^{*}$ has the following properties:

(a) $K$ has no convergent sequences.

(b) Nonempty open sets and infinite closed sets in $K$ are uncountable.

(c) If $A$ is as in the statement of Lemma 9(e) we may assume that $\mu_{n}(A)$ $=\sum_{m \in b} \mu_{n}\left(A_{m}^{\prime}\right)$ for each $n \in a$.

(d) $C(K)$ is a Grothendieck space.

(e) If $x$ is a point of $K$ then $K-\{x\}$ is $C^{*}$-embedded in $K$.

Proof. For (a) suppose $\left(x_{n}: n \in N\right)$ is a convergent sequence in $K$. Consider the Dirac measures $\mu_{n}$ concentrated at $x_{n}, A_{n}$ some sets around $x_{n}$, and $\left(\alpha_{n}: n \in N\right)$ satisfying the hypothesis of Lemma 9. We have $\varepsilon=1 / 2$. Now let $A$ and $b \subseteq a \subseteq N$ be as in (e)-(g) of Lemma 9. We conclude that $A \in x_{n}$ if $n \in b$ and $A \notin x_{n}$ if $n \in b-a$. So $\left(x_{n}: n \in N\right)$ is not convergent.

Clause (b) is a consequence of a general fact that compact countable spaces are metrizable and so have convergent sequences if they are infinite. Since by Lemma $9(\mathrm{C}), K$ has no isolated points, and compact spaces are normal, open sets contain infinite closed sets and so they are uncountable.

To prove (c) let $\left\{N_{\xi}: \xi<\omega_{1}\right\}$ be a family of infinite subsets of $N$ such that $N_{\xi} \cap N_{\xi^{\prime}}$ is finite whenever $\xi \neq \xi^{\prime}$. We may apply Lemma 9(a)-(g) to $\left\{A_{n}: n \in N\right\}$ for $N=N_{\xi}$ for each $\xi<\omega_{1}$, obtaining $b_{\xi} \subseteq a_{\xi} \subseteq N_{\xi}$, $A_{n}^{\xi}$ and $A_{\xi}$ like in Lemma 9(b). It will be sufficient to note that we have (c) for one of these families $\left\{A_{n}^{\xi}: n \in b_{\xi}\right\}$. Note that for any sequence of 
pairwise disjoint elements $A_{m}$ the set $\overline{\bigcup_{m \in N}\left[A_{m}\right]}-\bigcup_{m \in N}\left[A_{m}\right]$ is exactly the set of all those points of $K$ every neighbourhood of which intersects infinitely many sets $\left[A_{m}\right]$. In particular removing from the family of $A_{m}$ 's finitely many elements does not affect the set. Consequently,

$$
\begin{aligned}
& \left(\overline{\bigcup_{m \in b_{\xi}}\left[A_{m}^{\xi}\right]}-\bigcup_{m \in b_{\xi}}\left[A_{m}^{\xi}\right]\right) \cap\left(\overline{\bigcup_{m \in b_{\xi^{\prime}}}\left[A_{m}^{\xi^{\prime}}\right]}-\bigcup_{m \in b_{\xi^{\prime}}}\left[A_{m}^{\xi^{\prime}}\right]\right) \\
& =\left(\overline{\bigcup_{m \in b_{\xi}-b_{\xi^{\prime}}}\left[A_{m}^{\xi}\right]}-\bigcup_{m \in b_{\xi}-b_{\xi^{\prime}}}\left[A_{m}^{\xi}\right]\right) \cap\left(\overline{\bigcup_{m \in b_{\xi^{\prime}}-b_{\xi}}\left[A_{m}^{\xi^{\prime}}\right]}-\bigcup_{m \in b_{\xi^{\prime}}-b_{\xi}}\left[A_{m}^{\xi^{\prime}}\right]\right) \\
& \subseteq\left(A_{\xi}-\bigcup_{m \in b_{\xi} \cap b_{\xi^{\prime}}}\left[A_{m}\right]\right) \cap\left(A_{\xi^{\prime}}-\bigcup_{m \in b_{\xi} \cap b_{\xi^{\prime}}}\left[A_{m}\right]\right)=\emptyset .
\end{aligned}
$$

So the sets above are pairwise disjoint, and hence one of them is null with respect to all the measures. For this set we have

$$
\begin{aligned}
\mu_{n}\left(A_{\xi}\right) & =\mu_{n}\left(\bigvee_{m \in b_{\xi}} A_{m}^{\xi}\right)=\mu_{n}\left(\overline{\bigcup_{m \in b_{\xi}}\left[A_{m}^{\xi}\right]}\right) \\
& =\sum_{m \in b_{\xi}} \mu_{n}\left(A_{m}^{\xi}\right)+\mu_{n}\left(\overline{\bigcup_{m \in b_{\xi}}\left[A_{m}^{\xi}\right]}-\bigcup_{m \in b_{\xi}}\left[A_{m}^{\xi}\right]\right)=\sum_{m \in b_{\xi}} \mu_{n}\left(A_{m}^{\xi}\right),
\end{aligned}
$$

which completes the proof of (c).

To see that $C(K)$ is Grothendieck, i.e., that convergent sequences of Radon measures in the ( weak $\left.^{*}\right)$ topology induced by $C(K)$ are also convergent in the (weak) topology induced by the dual to the space of measures, assume that $\left(\mu_{n}\right)_{n \in N}$ is not weakly convergent but is weak* convergent to $\mu$.

If $\left(\mu_{n}\right)_{n \in N}$ were relatively weakly compact, but not a convergent sequence, by the Eberlein-Šmulian theorem it would be relatively sequentially weakly compact and there would exist disjoint infinite $c_{0}, c_{1} \subseteq N$ such that $\left(\mu_{n}\right)_{n \in c_{i}}$ would be weakly convergent to $\nu_{i}$ for $i \in\{0,1\}$, where $\nu_{0} \neq \nu_{1}$. But weakly convergent sequences are weak* convergent, a contradiction.

So we may assume that $\left(\mu_{n}\right)_{n \in N}$ is not relatively weakly compact; thus as a bounded sequence (applying the principle of uniform boundedness, since it is weak* convergent), by the Dieudonné-Grothendieck theorem ([Di, VII.14]) without loss of generality we may assume that there is a sequence $\left(A_{n}\right)_{n \in N}$ of pairwise disjoint clopen subsets of $K$ such that $\left|\mu_{n}\left(A_{n}\right)\right|>\varepsilon$. The points $x_{\alpha_{n}}$ could be chosen artificially so that the hypothesis of Lemma 9 (B) is satisfied. Let $A \in \mathcal{A}^{*}$ be as in Lemma 9(B)(e); we may assume that $A$ satisfies (c) of Proposition 10. By Lemma 9(B)(f), $\chi_{A}$ separates $\left(\mu_{n}\right)_{n \in b}$ from $\left(\mu_{n}\right)_{n \in a-b}$ in the weak* topology, i.e., $\left(\mu_{n}\right)_{n \in N}$ is not convergent in the weak* topology.

To prove (e) recall that $X \subseteq Y$ is $C^{*}$-embedded in $Y$ if and only if any bounded continuous function on $X$ extends to a continuous function on $X$. 
It is easy to see that if $K-\{x\}$ is not $C^{*}$-embedded in $K$, then there is a continuous function $f: K-\{x\} \rightarrow[0,1]$ and reals $0<r_{1}<r_{2}<1$ such that $x \in \overline{f^{-1}\left[\left[0, r_{1}\right)\right]} \cap \overline{f^{-1}\left[\left(r_{2}, 1\right]\right]}$. But $x$ can be the unique point of this intersection, which contradicts Lemma $9(\mathrm{C})$.

Definition 11 ([Ko]). Suppose that $K$ is a totally disconnected compact space. An operator $T: C(K) \rightarrow C(K)$ is called a weak multiplier if and only if for every sequence $\left(\left[A_{n}\right]: n \in N\right)$ of pairwise disjoint clopen subsets of $K$ we have

$$
\lim _{n \rightarrow \infty}\left\|T\left(\chi_{\left[A_{n}\right]}\right) \mid\left(K-\left[A_{n}\right]\right)\right\|=0 .
$$

Lemma 12. Every operator $T: C(K) \rightarrow C(K)$ is a weak multiplier.

Proof. This is very much like Lemma 3.2 of [Ko]. Suppose that a bounded linear operator $T: C(K) \rightarrow C(K)$ is not a weak multiplier, i.e., that there exist a bounded sequence of pairwise disjoint clopen sets $\left(\left[A_{n}\right]: n \in N\right)$ such that there is $\varepsilon>0$ and points $x_{n} \in K$ such that $x_{n} \notin\left[A_{n}\right]$ and $\left|T\left(\chi_{\left[A_{n}\right]}\right)\left(x_{n}\right)\right|>\varepsilon$ for infinitely many $n$. Since finite sums of the characteristic functions of $\left[A_{n}\right]$ 's are of norm one, if $x_{n}$ were constant for infinitely many $n$, we would get a contradiction with the fact that $T$ is bounded. Thus, we may assume without loss of generality that $\left|T\left(\chi_{\left[A_{n}\right]}\right)\left(x_{n}\right)\right|>\varepsilon$ holds for all $n \in N$ and that $x_{n}=x_{\xi_{n}}$ for some sequence $\left(\xi_{n}: n \in N\right)$ of distinct countable ordinals, since $\left\{x_{\xi}: \xi \in \omega_{1}\right\}$ is dense in $K$. By going to a subsequence we can assume that $\left(x_{\xi_{n}}: n \in N\right)$ is relatively discrete.

We may also assume without loss of generality that the points $x_{\xi_{n}}$ are not in the sets $\left[A_{m}\right]$ for $n, m \in N$ : if there is an $n_{0}$ such that $x_{\xi_{n}} \in\left[A_{n_{0}}\right]$ for $n$ 's from an infinite set $M \subseteq N$, we may consider $M-\left\{n_{0}\right\}$ and use the disjointness of $A_{n}$ 's. Otherwise, one can construct by induction an infinite set of indices as required. Thus $\left(A_{n}: n \in N\right)$ and $\left(x_{\xi_{n}}: n \in N\right)$ satisfy (a) and (b) of Lemma 9.

Let $\mu_{n}$ be the Radon measure on $K$ which corresponds (see [Se, §18]), by the Riesz representation theorem, to the linear bounded functional $\phi_{n}$ on $C(K)$ given by the relation

$$
\phi_{n}(f)=T(f)\left(x_{\xi_{n}}\right)=\int_{K} f d \mu_{n}
$$

which holds for all $f \in C(K)$, i.e., $\mu_{n}=T^{*}\left(\delta_{x_{\xi_{n}}}\right)$. In particular, we have $\left|\mu_{n}\left(\left[A_{n}\right]\right)\right|>\varepsilon$. We will be abusing notation and will use the same symbol $\mu_{n}$ for the measure on the Boolean algebra defined by $\mu_{n}(A)=\mu_{n}([A])$ for any $A \in \mathcal{A}^{*}$. Thus (B)(c), (d) of Lemma 9 are satisfied as well. So we may apply the lemma obtaining $b \subseteq a \subseteq N, \delta>0$ and a supremum $A$ and $A_{n}$ 's 
like in Lemma 9. By Proposition 10(c) we may assume that

$$
\mu_{n}(A)=\sum_{m \in b} \mu_{n}\left(A_{m}\right)
$$

Since $[A]$ is clopen, $\chi_{[A]}$ is continuous; let us analyze $T\left(\chi_{[A]}\right)$. By $(*)$ and Lemma $9(\mathrm{~B})(\mathrm{f})$ we conclude that if $n \in b$ we have

$$
\begin{aligned}
\left|T\left(\chi_{[A]}\right)\left(x_{\xi_{n}}\right)\right| & =\left|\mu_{n}(A)\right|=\left|\mu_{n}\left(A_{n}\right)+\sum\left\{\mu_{n}\left(A_{m}\right): m \neq n, m \in b\right\}\right| \\
\geq \varepsilon-\varepsilon / 3 & =2 \varepsilon / 3 .
\end{aligned}
$$

But if $n \in a-b$, we have

$$
\left|T\left(\chi_{[A]}\right)\left(x_{\xi_{n}}\right)\right|=\left|\mu_{n}(A)\right|=\left|\sum\left\{\mu_{n}\left(A_{m}\right): m \in b\right\}\right| \leq \varepsilon / 3 .
$$

Since $T\left(\chi_{[A]}\right)$ is continuous, we have that the closures of the sets $\left\{x_{\xi_{n}}\right.$ : $n \in b\}$ and $\left\{x_{\xi_{n}}: a-b\right\}$ are disjoint, hence can be separated by a clopen set; but this contradicts Lemma 9(B)(g) and completes the proof of Lemma 12.

Corollary 13. Any operator on $C(K)$ is of the form $g I+S$, where $g \in C(K)$ and $S$ is weakly compact or equivalently strictly singular.

Proof. It is proved in $[\mathrm{Ko}, 2.7]$ that a $C(K)$ space where all operators are weak multipliers and $K-\{x\}$ is $C^{*}$-embedded in $K$ for every $x \in K$ has the property that all operators are of the form as in the corollary. The fact that weakly compact operators on $C(K)$ 's are exactly strictly singular operators (i.e., those which are not an isomorphism when restricted to any infinite-dimensional space) was first proved in [Pe].

Corollary 14. Any complemented subspace of $C(K)$ is finite-dimensional or of density $\kappa$.

Proof. Suppose that $X \subseteq C(K)$ is an infinite-dimensional complemented subspace of $C(K)$ and let $Y$ be its complement. It is well known (see [Se]) that this is equivalent to the existence of a bounded projection $P: C(K) \rightarrow X$ onto $X$ and that $1-P$ is a projection onto $Y$. A projection is an operator such that $P^{2}=P$. Applying the previous results about decompositions of operators on $C(K)$ to the last equation we obtain a continuous function $g$ and a strictly singular operator $S$ such that $P=g I+S$ and

$$
g^{2} I+S g+g S+S^{2}=g I+S .
$$

That is, multiplication by $g^{2}-g$ is strictly singular, which means that $g^{2}-g$ cannot be nonzero over any infinite open set, that is, $g^{2}-g$ is always 0 , as our $K$ has no isolated points. This implies that $g$ assumes only values 1 or 0 , i.e., $g=\chi_{A}$ for some clopen $A$. If $A$ is empty, then $P$ is strictly singular and $X$ finite-dimensional. Thus $A$ is nonempty, hence by Lemma $9(\mathrm{~A}), C(A)$, the range of $g I$, has density $\kappa$. 
Now we need to note that as $K$ has a dense subset of cardinality $\omega_{1}$, the ranges of weakly compact operators on $C(K)$ have density at most $\omega_{1}$. Take any $Z \subseteq C(K)$ which is weakly compact and observe that $\phi: Z \rightarrow$ $R^{\omega_{1}}$ defined by $\phi(f)\left(x_{\xi}\right)$ is continuous with respect to weak and product topologies. It is also one-to-one, hence a homeomorphism, since $Z$ is compact in the weak topology. But $R^{\omega_{1}}$ has weight $\omega_{1}$ and hence $\phi[Z]$ has weight and so density $\omega_{1}$. As the range of $S$ is a countable union of weakly compact sets, it has density less than or equal to $\omega_{1}$.

Finally, as $g I=P-S$, the density of the range of $P$ must be $\kappa$.

REMARK. As in $[\mathrm{Ko}]$ one could prove several other interesting properties of $C(K)$.

\section{References}

[AL] D. Amir and J. Lindenstrauss, The structure of weakly compact sets in Banach spaces, Ann. of Math. 88 (1968), 35-46.

[De] K. Devlin, Constructibility, Perspect. Math. Logic, Springer, Berlin, 1984.

[Di] J. Diestel, Sequences and Series in Banach Spaces, Springer, 1984.

[DS] N. Dunford and J. Schwartz, Linear Operators. Part I, General Theory, Interscience Publ., New York, fourth printing, 1967.

[Fe] V. V. Fedorchuk, On the cardinality of hereditarily separable compact Hausdorff spaces, Soviet Math. Dokl. 16 (1975), 651-655.

[Gu] S. Gul'ko, On complemented subspaces of Banach spaces of the weight continuum, Ekstremal'nye Zadachi Teor. Funkts. 8 (1990), 34-41.

[H] F. Hausdorff, Über zwei Sätze von G. Fichtenholz und L. Kantorovitch, Studia Math. 6 (1936), 18-19.

[Ha] R. Haydon, A non-reflexive Grothendieck space that does not contain $l_{\infty}$, Israel J. Math. 40 (1981), 65-73.

[HM] S. Heinrich and P. Mankiewicz, Applications of ultrapowers to the uniform and Lipschitz classification of Banach spaces, Studia Math. 73 (1982), 225-251.

[Je] T. Jech, Set Theory, the third millennium edition, revised and expanded, Springer Monogr. Math., Springer, Berlin, 2003.

[JL] W. Johnson and J. Lindenstrauss, Some remarks on weakly compactly generated Banach spaces, Israel J. Math. 17 (1974), 219-230; Correction, ibid. 32 (1979), 382-383.

[Ka] A. Kanamori, The Higher Infinite. Large Cardinals in Set Theory from Their Beginnings, Perspect. Math. Logic, Springer, Berlin, 1994.

[Ko] P. Koszmider, Banach spaces of continuous functions with few operators, Math. Ann. 330 (2004), 151-183.

[Ku] K. Kunen, Set Theory, An Introduction to Independence Proofs, North-Holland, 1980.

[Pe] A. Pełczyński, On strictly singular and strictly cosingular operators. I. Strictly singular and strictly cosingular operators in $C(S)$-spaces, Bull. Acad. Polon. Sci. 13 (1965), 31-37.

[Pl] A. Plichko, On bases and complements in non-separable Banach spaces, Sibirsk. Math. J. 25 (1984), 155-162. 
[PY] A. Plichko and D. Yost, Complemented and uncomplemented subspaces of Banach spaces, Extracta Math. 15 (2000), 335-371.

[Ro] H. Rosenthal, On relatively disjoint families of measures with some applications to Banach space theory, Studia Math. 37 (1970), 13-36.

[Se] Z. Semadeni, Banach Spaces of Continuous Functions, PWN, 1971.

[Si] R. Sikorski, Boolean Algebras, Springer, 1964.

[Ta] M. Talagrand, Un nouveau $\mathcal{C}(K)$ qui possède la propriété de Grothendieck, Israel J. Math. 37 (1980), 181-191.

[Ve1] D. Velleman, Simplified morasses, J. Symbolic Logic 49 (1984), 257-271.

[Ve2] —, Simplified gap-2 morasses, Ann. Pure Appl. Logic 34 (1987), 171-208.

Departamento de Matemática

Universidade de São Paulo

Caixa Postal 66281

São Paulo, SP

CEP: 05315-970, Brasil

E-mail: piotr@ime.usp.br

URL: http://www.ime.usp.br/ ${ }^{\sim}$ piotr/stronamat.html

Received June 3, 2003

Revised version November 16, 2004 